\section{Interpretation of the Continuous Spectra of Comets}

Cometary dust, as well as being one of the basic features of comets, is of importance in several major problems of the solar system: it is most nearly representative of solid particles in the primordial solar nebula ${ }^{1}$; it is a contributor to zodiacal light ${ }^{2}$; and, although Harwit ${ }^{3}$ has questioned its effectiveness, it plays some part in processes in interplanetary space ${ }^{4}$. Information concerning the nature of the dust can be obtained at present only by an analysis of scattered sunlight in the heads and tails of comets.

Mie scattering calculations for single particles and for cumulative scattering functions ${ }^{5}$ were carried out on a computer at Goddard Space Flight Center ${ }^{6}$. These results were applied to measurements of the continuum in two ways.

Quantitative calculations were carried out for size distributions of the form

$$
f(a)=C a^{\alpha}, a_{1} \leqslant a \leqslant a_{2}
$$

where $a_{1}$ and $a_{2}$ are minimum and maximum radii and $\alpha$ took on integral values between -4 and 2 . Five size ranges were used for each distribution:

$$
\begin{aligned}
& \text { case }(a) 5 \times 10^{-7} \leqslant a \leqslant 1.5 \times 10^{-4} \mathrm{~cm} \\
& \text { (b) } 5 \times 10^{-7} \leqslant a \leqslant 5 \times 10^{-5} \\
& \text { (c) } 1.5 \times 10^{-6} \leqslant a \leqslant 1.5 \times 10^{-4} \\
& \text { (d) } 5 \times 10^{-6} \leqslant a \leqslant 1.5 \times 10^{-4} \\
& \text { (e) } 5 \times 10^{-5} \leqslant a \leqslant 1.5 \times 10^{-4} \mathrm{~cm}
\end{aligned}
$$

Both intensity and degree of polarization were computed and compared with observations of the comets 1957 III and $1957 \mathrm{~V}$ (ref. 7). They follow the same procedure as used previously ${ }^{8}$.

Cumulative scattering functions ${ }^{5}$ are defined as

$$
F_{j}(X)=\int_{0}^{X} i_{j}(x) \mathrm{d} x
$$

where $X=2 \pi a_{\max } / \lambda$ and runs from 0.1 to 25 in steps of 0.1 and the subscript $j$ represents either of the two polarization components. These functions were used to obtain qualitative characteristics of scattering by dielectric and iron spheres. In this analysis there was no restriction on the shape of the particle distribution nor of the size range for $X$ less than 25. Comparisons were made between these data and observations of comets 1957 III and $1957 \mathrm{~V}$.

In each investigation it was found that the observed degree of polarization, about 20 per cent, was too low to be caused by a distribution of iron spheres as proposed by Liller. The small variation of polarization with wavelength also supported this. We conclude that iron grains can make only a small contribution to the scattering in comets. The observations can be explained, however, by the presence of a cloud of dielectric particles.

Some preliminary calculations indicate that scattering from carbon grains will resemble scattering from iron. For scattering angles close to $90^{\circ}$, calculations for spheres agree rather closely with measurements on non-spheroidal particles ${ }^{5}$.

Infra-red measurements were made on the comet Ikeya-Seki ${ }^{10}$ by Beklin and Westphal in four wavelength intervals: $1 \cdot 5-1 \cdot 8 \mu, 2 \cdot 0-2 \cdot 4 \mu, 3 \cdot 0-3 \cdot 8 \mu$ and $8 \cdot 4-$ $13.5 \mu$. Infra-red emissivities were derived on the assumption that molecular emission could be neglected. The calculations were corrected for scattered sunlight. Spectra in the $2 \cdot 2 \mu$ region are quoted as showing less than 10 per cent line emission. A survey of predicted infra-red emissions in comets ${ }^{11}$ shows that the $3 \cdot 4 \mu$ region should be rich in molecular emission, much more so than the $2 \cdot 2 \mu$ region. The $10 \mu$ region was not examined nor were atomic lines in the infra-red. Without spectroscopic study, it is not safe to assume that the measurements of the $3 \cdot 4 \mu$ and $10 \mu$ filters are not affected by atomic or molecular emis- sions. It is well known that, to make intensity measurements of the continuum in the visible, great care is required in the choice of wavelength regions passed by the filters.

In seeking to match their deduced emissivities Beklin and Westphal ${ }^{10}$ used the optical properties of bulk material and suggested that iron gave a satisfactory fit. Cometary grains cannot be larger than a few microns radius without requiring excessive mass ${ }^{12}$. A similar limiting radius is required for the grains to be carried along by the escaping molecules ${ }^{13}$. For sizes less than the limiting radius the emissivity depends on the ratio radius/wavelength as well as index of refraction. Thus, even for the emissivities derived by Beklin and Westphal, it is not valid to say that iron is satisfactory without determining the emissivities of small grains.

Weinberg ${ }^{14}$ has obtained extensive measurements of the intensity and polarization of the continuum of the comet Ikeya-Seki at 8 wavelengths throughout the visible spectrum. The analysis of this material will be of great value for the interpretation of the continuum of comets. The difficulty of making a unique determination of the nature of a cloud of scattering particles from optical observations is well known. Measurements of intensity and polarization over a wide wavelength interval and over as large a range of scattering angles as possible are necessary for interpreting the continuum of comets.

\section{B. Donn}

Goddard Space Flight Center,

Greenbelt, Maryland.

\section{R. S. POWELL*}

Melpar Incorporated,

Falls Church, Virginia.

Institute d'Astrophysique,

\section{Remy-Battiau}

Université de Liège,

Cointe-Sclessin, Belgium.

* Supported in part by the U.S. National Aeronantics and Space Administration.

Received December $9,1966$.

1 Donn, B. D., Icarus, 2, 396 (1963).

2 Whipple, F. L., Astrophys. J., 121, 750 (1966).

${ }^{3}$ Harwit, M., J. Geophys. Res., 68, 2171 (1963).

'Belton, M. J. S., Astro. J., 70, 451 (1965). ${ }^{5}$ Donn, B. D., and Powell, R. S., Proc. Interdisc. Conf. on Electromag.

${ }^{6}$ Powell, R. S., Michels, T., Burley, J., and Donn, B. D. (to be published).

' Vainu Bappu, M. K., and Sinvhal, S. D., Mon. Not. Roy. Astro. Soc.,120, 152 $(\mathbf{1 9 6 0 )}$.

${ }^{8}$ Remy-Battiau, L., Bull. Acad. Roy. Belg. (Classe des Sci.), 5 Ser., 50, 74 (1964).

${ }^{\circ}$ Liller, W., Astrophys. J.,132, 867 (1960).

${ }^{10}$ Beklin, E. E., and Westphal, J. S., Astrophys. J., 145, 445 (1966).

Swings, P., in Space Age Astronomy (edit. by Deutsch, S. J., and Klempeser, W. B.), 370 (Academic Press, New York, 1962).

12 Swings, P., in Proc. Interdisc. Conf. on Electromag. Scattering (edit. by Kerker, M.), 159 (Pergamon Press, Oxford, 1963).

${ }^{13}$ Huebner, W. F., and Wiegert, A., Zeit. Astrophys., 64, 185 (1966).

13 Weinberg, J. L., Astro. J. (1966), paper presented at 122nd meeting, Amer. Astro. Soc., Cornell Univ. (July 1966).

\section{Secular Movement of the Zone of Auroral Blackout}

THE magnetic dip pole in 1922 and 1942 was close to the position $71^{\circ} \mathrm{N} .97^{\circ} \mathrm{W}$. whereas in 1955 and 1965 it was near $76^{\circ} \mathrm{N} .101^{\circ} \mathrm{W} .{ }^{1}$. A test has been made to find whether this movement was accompanied by changes in the rate of occurrence of radio blackout near Inverness $\left(57.5^{\circ} \mathrm{N}\right.$., $4^{\circ} \mathrm{W}$.) using the $f_{\min }$ tables from the vertical incidence data taken in this area between 1942 and 1963. The ionograms are reasonably comparable for the period. Data for months on which the number of blackout entries $(B)$ were abnormally large because of low critical frequencies at night were rejected. Independent studies were made for epochs near solar minimum, 1942-43 and 1962-63, and near solar maximum, 1946-47 and 1957-58, and significant differences 